\title{
EXPReS SA2: Network provision for a global e-VLBI array
}

\section{Francisco Colomer*}

Observatorio Astronómico Nacional, Apartado 112, E-28803 Alcalá de Henares, Spain

E-mail: f.colomer@oan.es

Using high-speed communication networks, we wish to create a distributed, astronomical instrument of a continental and inter-continental scale - a Very Long Baseline Interferometer (VLBI) operating in real time, and connecting together some of the largest and most sensitive radio telescopes on the planet. The overall objective of EXPReS, is to create a production-level 'electronic' VLBI (e-VLBI) service, in which the radio telescopes are reliably connected to the central supercomputer at JIVE in the Netherlands, via a high-speed optical fibre communication network, including the pan-European research network, GÉANT. The Specific Action (SA2) deals with the provision of the needed equipment and infrastructure (fibre optics connection) to allow the VLBI data from the participating telescopes to reach the correlator using GÉANT. We describe here the status of these last-mile connections. For more detailed information see:

http://www.jive.nl/dokuwiki/doku.php?id=expres:sa2

The 8th European VLBI Network Symposium

September 26-29, 2006

Toruń, Poland

*Coordinator of EXPReS SA2. 


\section{Status of the last-mile (local loop) construction}

\begin{tabular}{|c|c|c|c|c|}
\hline Telescope & Current BW & Expected BW & Year & Notes \\
\hline JIVE correlator & $4 \times 1$ Gbps & $16 \times 1 \mathrm{Gbps}$ & & connected \\
\hline WSRT (14x25m) & $1 \mathrm{Gbps}$ & & & connected \\
\hline Onsala $(20+25 m)$ & $1 \mathrm{Gbps}$ & $10 \mathrm{Gbps}$ & 2007 & connected \\
\hline Jodrell Bank (76m) & $1 \mathrm{Gbps}$ & $10 \mathrm{Gbps}$ & & connected \\
\hline Cambridge (32m) & $1 \mathrm{Gbps}$ & & & connected \\
\hline Toruń (32m) & $1 \mathrm{Gbps}$ & & & connected \\
\hline Metsähovi (14m) & $1 \mathrm{Gbps}$ & $10 \mathrm{Gbps}$ & 2007 & connected \\
\hline OAN-Yebes/CNIG-IGN (40m) & $0.1 \mathrm{Mbps}$ & 2,5 Gbps & 2007 & \\
\hline Effelsberg (100m) & 2 Mbps & $1 \mathrm{Gbps}$ & 2007 & \\
\hline Medicina (32m) & $1 \mathrm{Gbps}$ & & & connected \\
\hline Noto $(32 \mathrm{~m})$ & & & unknown & \\
\hline Sardinia $(64 m)$ & & $2,5 / 10 \mathrm{Gbps}$ & 2009 & \\
\hline Shanghai $(25 \mathrm{~m})$ & $100 \mathrm{Mbps}$ & $1 \mathrm{Gbps}$ & 2007 & \\
\hline Urumqi (25m) & & $1 \mathrm{Gbps}$ & 2007 & \\
\hline Miyun (50m) & & $1 \mathrm{Gbps}$ & 2007 & \\
\hline Yunnan (10m) & & $1 \mathrm{Gbps}$ & 2007 & \\
\hline VIRAC (32m) & & $1 \mathrm{Gbps}$ & in progress & \\
\hline Hartebeesthoek (26m) & & $1 \mathrm{Gbps}$ & unknown & \\
\hline Tigo $(6 \mathrm{~m})$ & $1-7 \mathrm{Mbps}$ & $64 \mathrm{Mbps}$ & & \\
\hline $\operatorname{Arecibo}(305 \mathrm{~m})$ & $<32 \mathrm{Mbps}$ & $1 \mathrm{Gbps}$ & 2007 & \\
\hline AARNET & & & unknown & \\
\hline ATNF (Parkes, Mopra, ATCA) & 1 Gbps (local) & 2 Gbps (to JIVE) & & \\
\hline
\end{tabular}

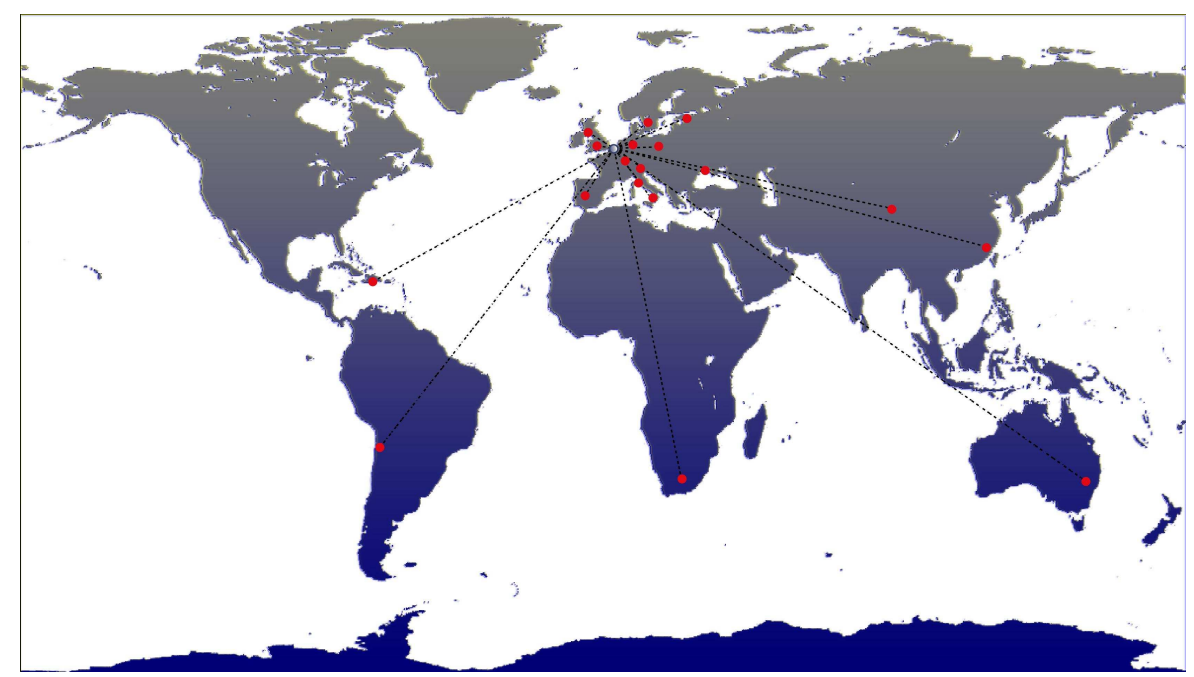

Figure 1: Map of the telescopes participating in EXPReS 

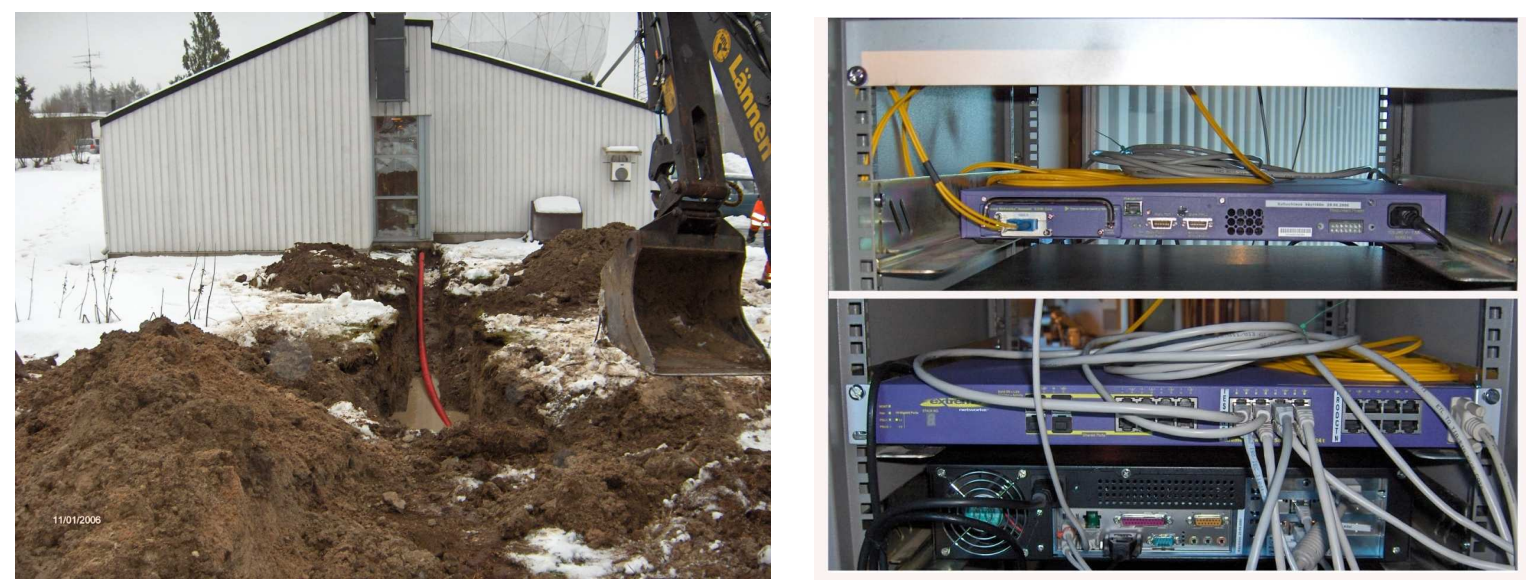

Figure 2: Last-mile fibre works (left) and equipment (right) at the 14-meter radio telescope in Metsähovi (Finland).
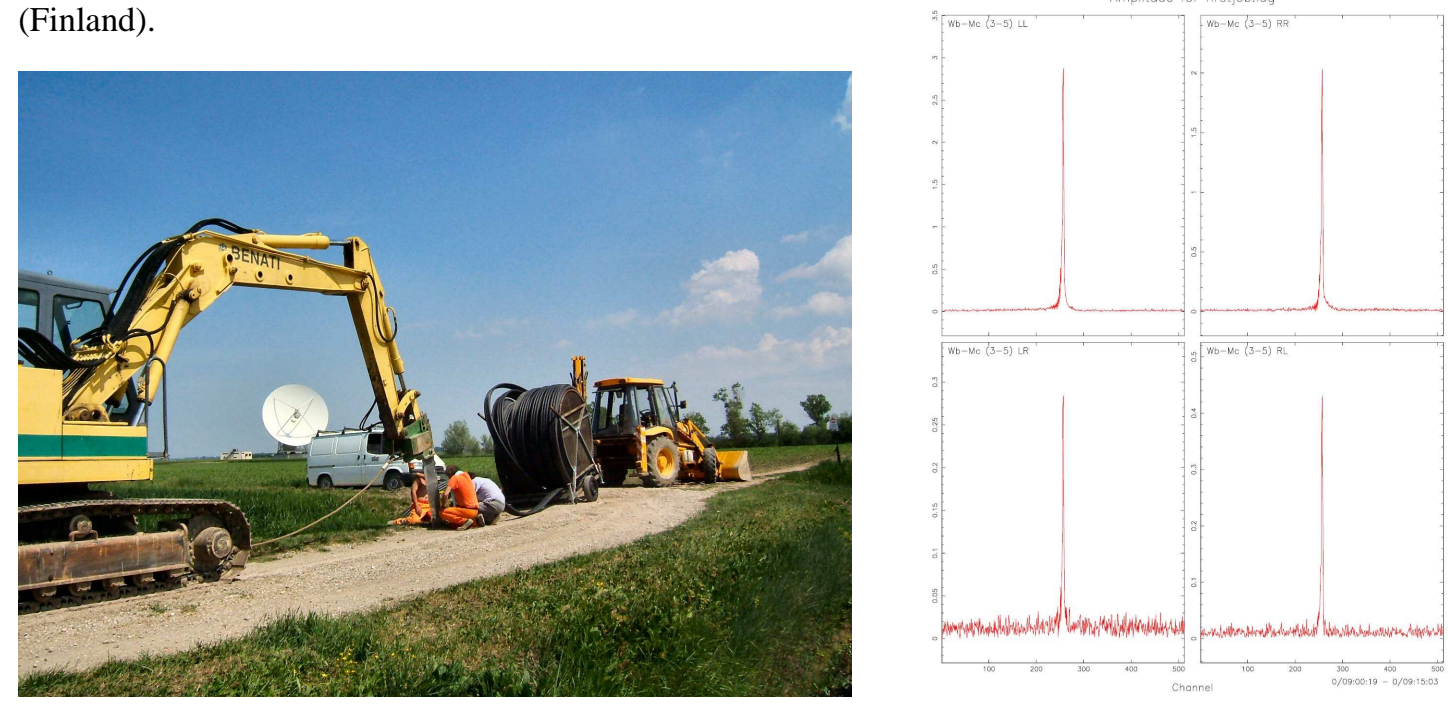

Figure 3: Left: Last-mile fibre works at the 32-meter radio telescope in Medicina (Italy). Right: First real-time interference fringes $(\mathrm{Mc}-\mathrm{Wb})$.
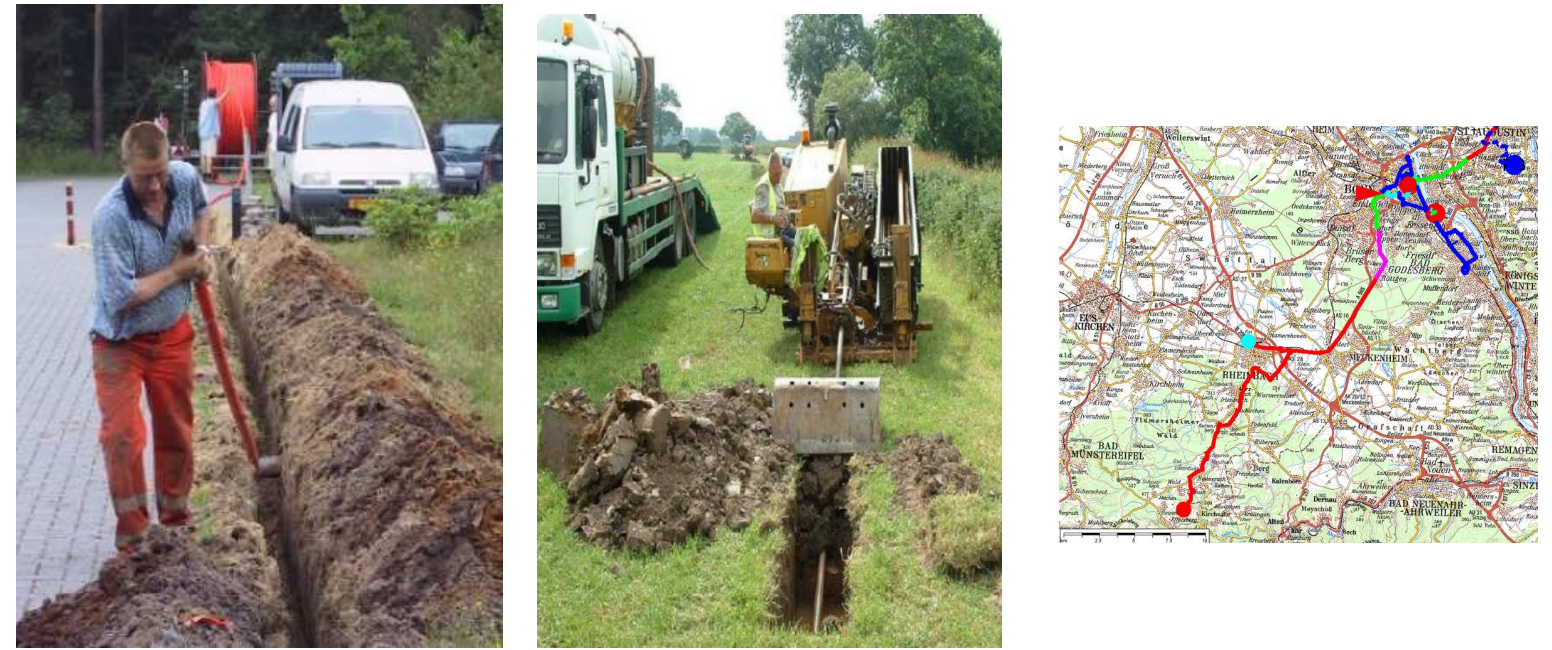

Figure 4: Last-mile fibre works at Westerbork (left), Jodrell Bank (center), and future line to Effelsberg (right). 